\title{
Impact of Ketone Salt Containing Supplement on Cardiorespiratory and Oxidative Stress Response in Firefighters Exercising in Personal Protective Equipment
}

\author{
Matthew John McAllister ${ }^{1,}{ }^{*}$, Angelia Maleah Holland ${ }^{2}$, Harish Chander ${ }^{3}$, Hunter Scott Waldman ${ }^{3}$, \\ JohnEric William Smith ${ }^{3}$ and Steven Allen Basham ${ }^{3}$ \\ ${ }^{1}$ Metabolic and Applied Physiology Lab, Department of Health and Human Performance, Texas State University, San Marcos, United States \\ ${ }^{2}$ Nutrition, Exercise and Stress Lab, Department of Kinesiology, Augusta University, Augusta, United States \\ ${ }^{3}$ Department of Kinesiology, Mississippi State University, Starkville, United States \\ "Corresponding author: Metabolic and Applied Physiology Lab, Department of Health and Human Performance, Texas State University, San Marcos, United States. Email: \\ mjm445@txstate.edu
}

Received 2018 July 19; Revised 2018 November 02; Accepted 2018 December 28.

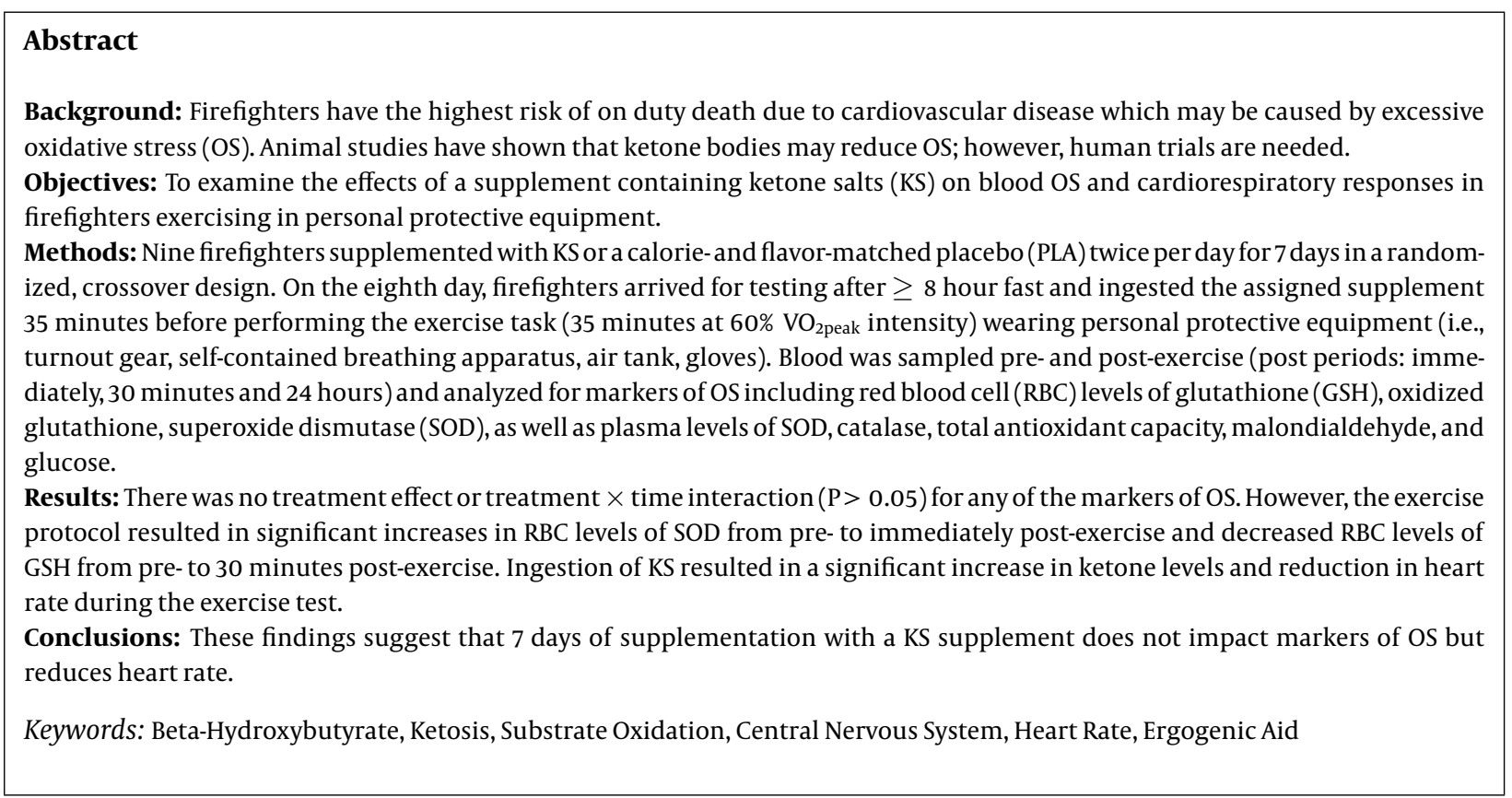

\section{Background}

Firefighters (FF) have the largest occupational-related risk of death due to cardiovascular disease (CVD) which may be caused by excessive oxidative stress (OS) (1). Firefighter occupational activities have been shown to produce increases in markers of OS due to increased metabolic demand from a variety of stressors (2-4). While acute exposure to moderate amounts of OS and inflammation is beneficial for stimulating favorable adaptations (5), chronic OS exposure may be a major contributor to increased CVD prevalence in $\mathrm{FF}(6)$.

OS is associated with reactive oxygen species (ROS) pro- duction that overwhelms endogenous antioxidants. Dietary interventions such as caloric-restriction (7) exogenous antioxidants (8), and a low-carbohydrate, ketogenic diet (9), have demonstrated improvements in endogenous antioxidant capacity and decreased OS. In addition, a ketogenic diet is associated with improved mitochondrial function via increased levels of uncoupling proteins (9), which results in less ROS production reducing OS (10-12).

Exogenous ketones may facilitate ketosis without following a ketogenic diet which involves extreme restrictions on carbohydrate intake $(13,14)$. It is not clear whether or not ketone bodies themselves are responsible for im- 
pacting biomarkers of OS, or if there are other mechanisms of adaptation. While the physiological responses to exogenous ketone supplementation and dietary interventions such as a ketognic diet are vastly different, previous authors $(13,14)$ have suggested exogenous ketone supplementation may produce benefits similar to the ketogenic diet such as reduced OS and inflammation $(13,14)$. Ketone bodies, beta-hydroxybutyrate $(\beta$-OHB) and acetoacetate (ACAC), may reduce ROS generation $(15,16)$ and facilitate antioxidant adaptations by acting as a histone deacetylase inhibitor (15). However, the majority of studies have been conducted in rodents $(12,13)$; therefore, human trials are needed.

\section{Objectives}

Thus, the purpose of this study was to investigate potential antioxidant adaptations resulting from ingesting a ketone salt (KS) supplement containing $\beta$-OHB in professional FF. Previous findings have also suggested that $\beta$-OHB can impact the sympathetic nervous system resulting in decreased heart rate (17). Therefore, a second aim of the study was to investigate the impact on cardiorespiratory responses to exercise. We hypothesized that the KS supplement would result in reduced markers of OS from the exercise protocol.

\section{Methods}

\subsection{Experimental Design}

This study utilized a randomized, double-blinded, placebo-controlled, cross-over design to investigate the effects of $\beta$-OHB supplementation on the OS and cardiorespiratory responses to exercise in FF wearing personal protective equipment (PPE). Participants provided written informed consent, completed a health history questionnaire, and subsequently completed three testing sessions. Session one included baseline measures of height (235D; Quick Medical, Issaquah, WA, USA), body mass (Defender 5000, Ohaus Corporation, Parsippany, NJ, USA), and mass of the PPE (i.e., self-contained breathing apparatus, jacket, pants, boots, gloves). A $\mathrm{VO}_{2 \text { peak }}$ test was also performed during session one. Following session one, subjects supplemented with either KS or a calorie- and flavor-matched placebo (PLA) for seven days. On the eighth day, subjects reported to the laboratory following at least an eight hour fast between the hours of 0500 and 0900 for session two. During session two, KS or PLA was ingested 35 minutes before a physical stress task (exercise in PPE at $60 \% \mathrm{VO}_{2 \text { peak }}$ for 35 minutes). Due to the rapid metabolism of exogenous ketones (13) a seven day washout period was incorporated between the seven day supplementation periods. Following the washout period, subjects then supplemented for seven days with the opposite treatment. Session three was performed on day eight (following seven days of supplementation with the opposite treatment) and involved the same physical stress task. Participants were asked to attempt to maintain consistent dietary and physical activity habits throughout the duration of the study.

\subsection{Subjects}

Ten male FF aged 18 - 39 were initially recruited from the local fire department to participate in the study. Nine FF completed all testing sessions and followed the supplemental protocol. Inclusion criteria included: (1) be an active duty firefighter, (2) meet the American College of Sports Medicine low-risk guidelines (18), (3) obtain at least 150 minutes of moderate-intensity aerobic activity or 60 minutes of vigorous intensity aerobic activity per week, (4) avoid consumption of dietary supplements that contain any antioxidants or ergogenic aids for two weeks prior to and throughout the duration of the study, and (5) refrain from caffeine and alcohol consumption 24 hours prior to the experimental testing sessions. The FF's work schedule involved 24 hours on duty, followed by 48 hours off duty. As part of the nature of this occupation, FF may not be able to maintain regular sleep and dietary habits. Thus, this could be viewed as a limitation that could impact results. However, the aim of the study was to involve professional FF to maximize potential application. The study was approved by the University's Institutional Review Board. Subject descriptive characteristics are shown in Table 1.

\begin{tabular}{|c|c|c|c|}
\hline Variable & Mean \pm SD & Minimum & Maximum \\
\hline Body mass, kg & $84.1 \pm 13.4$ & 61.5 & 113.5 \\
\hline Mass of gear, $\mathrm{kg}$ & $18.7 \pm 1.0$ & 17.2 & 19.9 \\
\hline Total mass, kg & $102.8 \pm 13.6$ & 80.9 & 133.2 \\
\hline Peak VO, $\mathrm{mL} / \mathrm{kg} / \mathrm{min}^{-1}$ & $40.0 \pm 3.7$ & 34.0 & 44.9 \\
\hline $60 \% \mathrm{VO}_{\text {2peak }}, \mathrm{mL} / \mathrm{kg} / \mathrm{min}^{-1}$ & $24.0 \pm 2.2$ & 20.4 & 26.9 \\
\hline
\end{tabular}

${ }^{a}$ Descriptive Characteristics Were Collected Before All Experimental Trials. Body Mass and the Mass of Firefighter Gear Were Recorded Separately.

\subsection{Testing Procedures}

Upon arrival to the laboratory, subjects were fitted with a heart rate monitor (FT1, Polar Electro Inc., Lake Success, NY, USA). Exercise testing took place on a Woodway treadmill (Waukesha, $\mathrm{WI}$ ). $\mathrm{VO}_{2 \text { peak }}$ testing was performed while subjects were wearing comfortable clothing (PPE was not worn due to safety concerns). Metabolic gases were collected using a MOXUS Modular metabolic system (AEI Technologies, Bastrop, TX). Each stage was three minutes in 
duration. Speed started at $4.0 \mathrm{~km} / \mathrm{h}$ with a $4 \%$ grade. Stages two through five maintained $4 \%$ grade and speed increased by $1.6 \mathrm{~km} / \mathrm{h}$ every stage until a speed of 10.4 $\mathrm{km} / \mathrm{h}$ was achieved. Every subsequent stage maintained the same speed and incorporated an increase in grade by $1 \%$. The test continued until volitional exhaustion. $\mathrm{VO}_{2 \text { peak }}$ was recorded as the highest $\mathrm{VO}_{2}$ values that averaged over the course of one minute. Subsequently, the weight of the gear was then added to the recorded body mass value to calculate each subject's relative $\mathrm{VO}_{2 \text { peak }}$ since the exercise protocol involved steady state exercise while wearing full gear. The exercise protocol for sessions two and three consisted of 35 minutes of steady state exercise at $60 \%$ of the calculated relative $\mathrm{VO}_{2 \text { peak }}$. Grade was set at $2 \%$ during sessions two and three. Speed was adjusted to keep $\mathrm{VO}_{2}$ at the appropriate 60\% target. During sessions two and three, visual analog scales for perceived mood, focus, energy, alertness, satiety, and hunger were collected thirty-five minutes before and fifteen minutes after exercise. Rating of perceived exertion (RPE) was collected at 5, 30, and 35 minutes after the start of exercise.

\subsection{Supplementation}

Participants were randomly assigned to the order in which they received the treatment or PLA. Randomization occurred using a random number generator and assigning treatments (A or B) to numbers that were generated (either 1 or 2 ). After randomization, 14 unlabeled packets coded "A" or "B" were provided to the subjects which contained either KS or PLA. Due to the rapid metabolism of the ingredients (13), the subjects were asked to ingest two packets per day (one in the morning, one in the early evening) for seven days. During sessions two and three, the supplement was ingested 35 minutes before the start of exercise. This protocol was designed based on previous findings that ketones can acutely reduce reactive oxygen species production and trigger antioxidant adaptations via acting as histone deacetylase inhibitors (19). A seven day wash-out period separated sessions two and three. Four FF had to respond to a building fire on the morning of the eighth day of supplementation, therefore, these four FF ingested their supplement for eight days and completed session two on the morning of the ninth day. Each packet was mixed with $0.23 \mathrm{~L}$ of water. Compliance was $100 \%$ and was recorded during each testing session based on the number of packets that were successfully ingested. The supplemental treatments included KS (Keto//OS® MAX ${ }^{\mathrm{TM}}$, Melissa TX) and each packet contained seven grams of KS, erythritol, L-Taurine, fermented L-Leucine, citric acid, natural flavor, vegetable juice color, stevia, xanthan gum, beta carotene, and approximately $920 \mathrm{mg}$ of sodium and PLA which was a maltodextrin powder that was color- and flavor-matched to KS. Both treatments contained 41 kcals. These methods are similar to a previous study (20) that used a KS mixture. Raw materials were tested for contaminants by the manufacturer.

\subsection{Blood Sampling and Analysis}

Twenty minutes after beverage ingestion, a finger prick was performed using a 26 gauge Dynarex $(1.8 \mathrm{~mm}$, Orangeburg, NY) self-withdrawing safety lancet. After cleaning with an alcohol swab, allowing to air dry, and wiping away the first blood droplet, 5 microliters $(\mu \mathrm{L})$ were analyzed for ketone (Precision Xtra, Abbott, Alameda, CA) levels. This is a method used in previous research to assess $\beta$-OHB levels from KS supplementation (20). In addition, blood samples were obtained just prior to the start of exercise, as well as immediately post exercise, as well as 30 minutes and 24 hours post exercise. Blood draws were performed with the participants in a supine position. A total of $12 \mathrm{~mL}$ was sampled at each draw from the antecubital vein. Blood was drawn into EDTA anticoagulant sealed vacutainers and centrifuged for 10 minutes at $2500 \mathrm{rpm}$ at $4^{\circ} \mathrm{C}$. Plasma was aliquoted and stored at $-80^{\circ} \mathrm{C}$. A volume of $200 \mu \mathrm{L}$ of erythrocytes (RBCs) were lysed in $800 \mu \mathrm{L}$ of ice cold HLPC water in duplicate microcentrifuge tubes and centrifuged at $10000 \mathrm{~g}$ for 15 minutes. The supernatant from one tube was aliquoted and stored at $-80^{\circ} \mathrm{C}$ for subsequent analysis of red blood cell (RBC) levels of superoxide dismutase (SOD). In addition, $400 \mu \mathrm{L}$ of the supernatant from the other microcentrifuge tube was mixed with $400 \mu \mathrm{L}$ of a metaphosphoric acid solution (Sigma Aldrich, St Louis, MO, USA) for deproteination. After the addition of metaphosphoric acid, the samples incubated for 5 minutes at room temperature and subsequently were centrifuged $10000 \mathrm{~g}$ for 5 minutes. The supernatant was collected and stored at $80^{\circ} \mathrm{C}$ for subsequent analysis of glutathione (GSH) and oxidized glutathione (GSSG) concentrations.

\subsubsection{Glucose}

Plasma glucose concentrations were analyzed in duplicate using a cuvette method glucose oxidase assay (Pointe Scientific, Canton, MI, USA). Samples and reagents were incubated at $37^{\circ} \mathrm{C}$ for ten minutes and absorbance at 500nm was read with a Pointe 180 II spectrophotometer (Pointe Scientific, Canton, MI, USA).

\subsubsection{Malondialdehyde}

Malondialdehyde (MDA) was analyzed in thawed plasma using a commercially available kit (Cayman Chemical, Ann Arbor, MI, USA). MDA is a marker of lipid peroxidation and in vitro reacts with a thiobarbituic acid in an aqueous solution and in the presence of heat at $45^{\circ} \mathrm{C}$ 
produces a pinkish color that absorbs light at $530-540$ $\mathrm{nm}$. Absorbance corresponds to the concentration of MDA. Plasma samples were thawed and assayed in duplicate. Absorbance was read at $540 \mathrm{~nm}$.

\subsubsection{Total Antioxidant Capacity}

Plasma total antioxidant capacity (TAC) was analyzed in units of Trolox equivalents (Cayman Chemical, Ann Arbor, MI, USA). Trolox is a water soluble vitamin E analog. This assay relies on the principle that antioxidants will inhibit the oxidation of 2, 2'-azino-di-3-ethylbenzthiazoline sulphonate induced by metmyoglobin. Under assay conditions, this reaction can be monitored by measuring the absorbance at 750 or $405 \mathrm{~nm}$ in such a way that the antioxidants cause suppression (thus an inverse relationship) of absorbance. Plasma was diluted 1:20 before assaying in duplicate samples and absorbance was read at $750 \mathrm{~nm}$ using an end point method.

\subsubsection{Superoxide Dismutase}

Superoxide dismutases (SODs) are metalloenzymes that facilitate the reduction of the superoxide anion $\left(\mathrm{O}_{2}{ }^{-}\right)$ to hydrogen peroxide $\left(\mathrm{H}_{2} \mathrm{O}_{2}\right)$. Plasma and $\mathrm{RBC}$ lysate was analyzed in duplicate samples for SOD concentrations (Cayman Chemical, Ann Arbor, MI, USA). This assay measures all three types of SOD (copper zinc, manganese, and iron). Xanthine oxidase was used as the pro-oxidant enzyme and tetrazolium salt was utilized for detection of radicals generated by xanthine oxidase and hypozantine. RBC lysate was diluted 1:100 before assaying. Plasma was diluted 1:5 before assaying. RBC lysate and plasma samples were analyzed in duplicate, and absorbance was read at $450 \mathrm{~nm}$.

\subsubsection{Catalase}

Plasma was analyzed for catalase (CAT) activity in duplicate samples (Cayman Chemical, Ann Arbor, MI, USA). CAT is an antioxidant enzyme that reduces hydrogen peroxide to $\mathrm{H}_{2} \mathrm{O}$. This assay uses methanol and hydrogen peroxide to impact CAT activity. The product of this reaction (formaldehyde) is measured colormetrically using 4-amino-3-hydrazino-5-mercapto-1,2,4-triazole as the chromogen. The addition of this substance facilitates a purple color which can be used to estimate CAT activity. Absorbance was read at $540 \mathrm{~nm}$ and CAT activity (nmol/min/ml) was calculated with formaldehyde concentrations.

\subsubsection{Glutathione}

$\mathrm{RBC}$ lysate samples were analyzed in duplicate samples for GSH and oxidized GSSG (Cayman Chemical, Ann Arbor,
MI, USA). GSH exists in the reduced form and is directly redox active as a reducing agent. GSSG can be recycled to replenish GSH in the presence of glutathione reductase. After sample deproteination with metaphosphoric acid, 50 $\mu \mathrm{L}$ of a $4 \mathrm{M}$ triethanolamine solution was added to samples and contained $531 \mu \mathrm{L}$ of triethanolamine (Sigma Aldrich, St Louis, MO, USA) and $469 \mu \mathrm{L}$ of HPLC grade water. The purpose of this step was to increase the $\mathrm{pH}$ of the sample. Samples were then diluted 1:10 before analysis of GSH and GSSG concentrations. Preparation of samples for GSSG analysis involved a $1 \mathrm{M}$ solution of 2-vinylpyridine which was created by mixing $108 \mu \mathrm{L}$ of 2-vinylpyridine (Sigma Aldrich, St Louis, MO, USA) with $892 \mu \mathrm{L}$ of reagent grade ethanol (Sigma Aldrich, St Louis, MO, USA). The standards provided in the kit were treated with the 2-vinylpyridine solution in the same way before performing the assay. Absorbance was read for GSH and GSSG at $405 \mathrm{~nm}$ using an end point method.

\subsection{Cardiorespiratory Variables}

Pulmonary variables $\mathrm{VO}_{2}, \mathrm{VCO}_{2}, \mathrm{~V}_{\mathrm{E}}$, respiratory exchange ratio (RER), and respiratory rate (RR) were averaged starting from minute 10 to minute 30 during exercise. Heart rate (HR) was recorded at minute 5, 10, 20, 30, and 35 after the start of exercise. RPE was measured at minute 5 , 30 and 35 after the start of exercise.

\subsection{Statistical Analysis}

All statistical procedures were conducted with SAS version 9.3 (Cary, NC, USA). Blood markers GSH, GSSG, SOD, MDA, TAC and HR were analyzed using a $2 \times 4$ (treatment $\times$ time) repeated measures ANOVA. Glucose and RPE were analyzed using a $2 \times 3$ (treatment $\times$ time) repeated measures ANOVA. Blood ketone levels and cardiorespiratory variables $\mathrm{VO}_{2}, \mathrm{VCO}_{2}, \mathrm{~V}_{\mathrm{E}}$, RER, and $\mathrm{RR}$ were analyzed with paired t tests. VAS data were analyzed via $2 \times 2$ (treatment $\mathrm{X}$ time) repeated measures ANOVA. Significance was set a priori $\mathrm{P}<0.05$. In the instance of a significant main effect, a Bonferroni correction was applied to compare means.

\section{Results}

\subsection{Blood Markers}

Blood ketone levels were significantly higher $(\mathrm{P}<$ 0.001) 30 minutes after beverage ingestion compared to baseline $(0.55 \mathrm{mmol} / \mathrm{L} \pm 0.133$ vs $0.08 \mathrm{mmol} / \mathrm{L} \pm 0.03$, respectively). There was no significant $(\mathrm{P}<0.05)$ treatment $\mathrm{X}$ time interaction for any blood variables. A summary of blood variables is shown in Table 2. Data are reported as mean \pm SE unless otherwise specified. 


\begin{tabular}{|c|c|c|c|c|c|c|c|c|}
\hline & \multicolumn{2}{|c|}{ PRE-EX } & \multicolumn{2}{|c|}{ POST-EX } & \multicolumn{2}{|c|}{$30 \mathrm{~min}$ POST } & \multicolumn{2}{|c|}{24 h POST } \\
\hline & PLA & $\beta$-ОНВ & PLA & $\beta$-онВ & PLA & $\beta$-ОНВ & PLA & $\beta$-онВ \\
\hline GSSG, $\mu \mathbf{M}$ & $39.3 \pm 11.3$ & $38.8 \pm 6.4$ & $37.0 \pm 11.2$ & $34.3 \pm 13.9$ & $37.8 \pm 9.5$ & $36.0 \pm 16.6$ & $43.2 \pm 9.8$ & $41.7 \pm 10.0$ \\
\hline PLASMA SOD, U/mL & $1.3 \pm 0.5$ & $1.6 \pm 0.7$ & $1.6 \pm 1.0$ & $1.3 \pm 0.3$ & $1.4 \pm 0.4$ & $1.2 \pm 0.4$ & $1.3 \pm 0.5$ & $1.8 \pm 1.0$ \\
\hline $\begin{array}{l}\text { CATALASE, } \\
\mathrm{nmol} / \mathrm{min} / \mathrm{mL}\end{array}$ & $29.2 \pm 11.5$ & $25.6 \pm 7.7$ & $26.9 \pm 9.0$ & $29.8 \pm 10.2$ & $27.0 \pm 9.0$ & $24.8 \pm 4.7$ & $25.5 \pm 10.7$ & $31.6 \pm 20.0$ \\
\hline MDA, $\mu \mathbf{M}$ & $4.9 \pm 1.8$ & $3.8 \pm 2.0$ & $4.8 \pm 2.5$ & $3.3 \pm 2.2$ & $3.3 \pm 1.3$ & $3.9 \pm 2.7$ & $3.4 \pm 2.0$ & $4.2 \pm 2.1$ \\
\hline TAC, $\mathbf{m M}$ & $0.6 \pm 0.3$ & $0.5 \pm 0.4$ & $0.5 \pm 0.2$ & $0.7 \pm 0.2$ & $0.5 \pm 0.2$ & $0.3 \pm 0.2$ & $0.4 \pm 0.3$ & $0.6 \pm 0.3$ \\
\hline $\operatorname{GLUCOSE}^{\mathrm{c}}, \mathbf{m g} / \mathbf{d L}$ & $88.2 \pm 18.4$ & $81.9 \pm 7.6$ & $83.8 \pm 7.1$ & $80.3 \pm 12.2$ & $73.0 \pm 9.9$ & $75.5 \pm 9.3$ & & \\
\hline
\end{tabular}

${ }^{a}$ Abbreviations: GSH, glutathione; GSSG, oxidized glutathione; MDA, malondialdehyde; RBC, red blood cell; SOD, superoxide dismutase; TAC, total antioxidant capacity.

a Values are represented as mean $\pm \mathrm{SD}$.

${ }^{\mathrm{b}}$ Blood samples were taken before exercise (PRE-EX), post exercise (POST-EX), as well as 30 minutes post exercise and 24 hours post exercise.

${ }^{\mathrm{c}}$ Denotes significant $(\mathrm{P}<0.05)$ main effect for time.

\subsubsection{GSH and GSSG}

In terms of RBC levels of GSH, there was no main effect for treatment $(\mathrm{F}=0.00, \mathrm{P}=0.956)$ and there was no treatment $\mathrm{X}$ time interaction $(\mathrm{F}=0.66, \mathrm{P}=0.578)$. There was a significant main effect for time for RBC levels of total GSH $\left(\mathrm{F}=3.45, \mathrm{P}=0.023, \eta_{\mathrm{p}}{ }^{2}=0.163\right)$. Total GSH was lower at preexercise compared to 30-minutes post-exercise $(\mathrm{P}=0.03)$. There was no difference between pre-exercise GSH and 24 hours post-exercise. Mean GSH concentrations are shown in Figure 1. Regarding RBC levels of GSSG, there was no significant treatment $\mathrm{X}$ time interaction $(\mathrm{F}=0.11, \mathrm{P}=0.953)$, and no significant main effect for treatment $(\mathrm{F}=0.29, \mathrm{P}=$ $0.595)$ or time $(F=1.66, P=0.186)$. Mean RBC levels of GSSG are shown in Table 2.

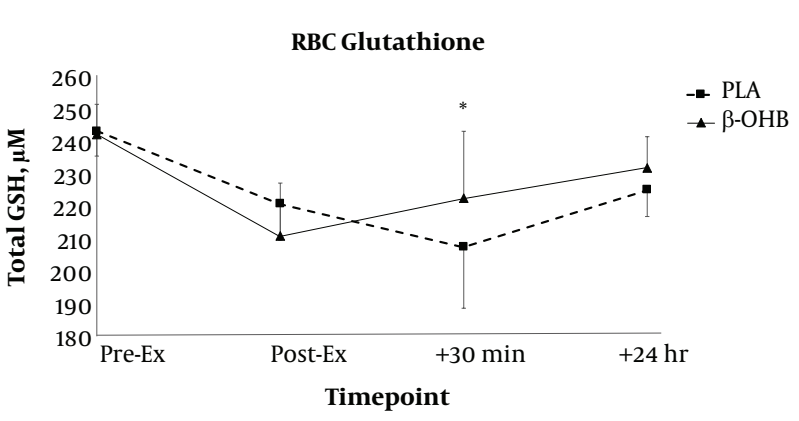

Figure 1. Changes in red blood cell (RBC) levels of glutathione (GSH) over time. Data are reported as mean \pm SE. Blood samples were taken before exercise (Pre-Ex), post exercise (Post-Ex), as well as 30 minutes post exercise ( +30 minutes) and 24 hours post exercise ( +24 hours). *denotes significant difference from all other time points. $\beta$-OHB = beta hydroxybutyrate, PLA = placebo.

\subsubsection{SOD}

In terms of RBC levels of SOD, there was no main effect for treatment $(\mathrm{F}=0.21, \mathrm{P}=0.646)$ and no treatment $\mathrm{X}$ time interaction $(\mathrm{F}=0.83, \mathrm{P}=0.482)$. There was a significant main effect for time for RBC levels of $\operatorname{SOD}(\mathrm{F}=2.87, \mathrm{P}=$ $\left.0.044, \eta_{\mathrm{p}}{ }^{2}=0.135\right)$. RBC levels of SOD were significantly elevated post exercise compared to other time points. Mean RBC levels of SOD are shown in Figure 2. For plasma levels of SOD, there were no main effects for treatment $(F=0.27$, $\mathrm{P}=0.603)$ or time $(\mathrm{F}=0.62, \mathrm{P}=0.602)$, and no treatment $\mathrm{X}$ time interaction $(\mathrm{F}=1.57, \mathrm{P}=0.207)$.

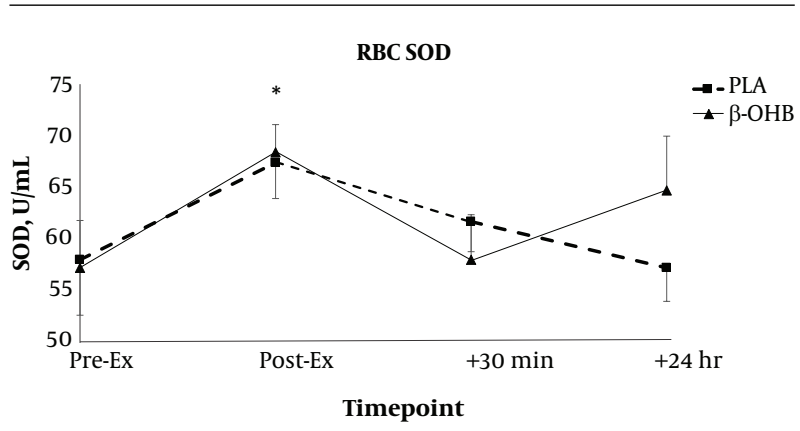

Figure 2. Changes in red blood cell (RBC) levels of superoxide dismutase (SOD) over time. Data are reported as mean \pm SE. Blood samples were taken before exercise(PreEx), post exercise (Post-Ex), as well as 30 minutes post exercise (+30 minutes) and 24 hours post exercise (+24 hours). *denotes significant difference from all other time points. $\beta$-OHB = beta hydroxybutyrate, PLA = placebo.

\subsubsection{Glucose}

There was a significant main effect for time for plasma glucose levels $\left(\mathrm{F}=5.54, \mathrm{P}=0.007, \eta_{\mathrm{p}}{ }^{2}=0.221\right)$. LSD post hoc revealed a significant decrease in blood glucose levels at 30-minutes post exercise compared to pre-exercise $(\mathrm{P}=$ $0.002)$ and immediately post exercise $(\mathrm{P}=0.026)$. There was no main effect for treatment $(\mathrm{F}=0.65, \mathrm{P}=0.426)$ and no treatment $\mathrm{X}$ time interaction $(\mathrm{F}=0.79, \mathrm{P}=0.461)$. Mean glucose levels are shown in Table 2. 


\subsubsection{TAC, CAT, MDA}

There was no significant main effect for time $(F=0.80$, $\mathrm{P}=0.499)$ or treatment $(\mathrm{F}=0.15, \mathrm{P}=0.697)$ and no treatment $X$ time interaction $(F=1.18, P=0.327)$ for plasma TAC. There was no significant main effect for time $(\mathrm{F}=0.35, \mathrm{P}=$ $0.791)$ or treatment $(\mathrm{F}=0.38, \mathrm{P}=0.540)$ and no treatment $\mathrm{X}$ time interaction $(\mathrm{F}=0.92, \mathrm{P}=0.439)$ for plasma CAT activity. Further, there was no significant main effect for time $(\mathrm{F}=0.42, \mathrm{P}=0.742)$ or treatment $(\mathrm{F}=0.35, \mathrm{P}=0.555)$ and no treatment $\mathrm{X}$ time interaction $(\mathrm{F}=1.34, \mathrm{P}=0.269)$ for plasma MDA. Mean TAC, CAT, and MDA levels are shown in Table 2.

\subsection{Cardiorespiratory Variables}

There was no significant difference between treatments in mean $\mathrm{VO}_{2}(\mathrm{P}=0.249), \mathrm{V}_{\mathrm{E}}(\mathrm{P}=0.361), \mathrm{RR}(\mathrm{P}=0.572)$, $\operatorname{RER}(\mathrm{P}=0.409)$, and $\mathrm{VCO}_{2}(\mathrm{P}=0.275)$. There was no significant treatment $\times$ time interaction for $\mathrm{HR}(\mathrm{F}=0.07, \mathrm{P}$ $=0.991)$. However, there was a main effect for time $(\mathrm{F}=$ 42.43, $\mathrm{P}<0.001)$ as well as treatment $(\mathrm{F}=5.44, \mathrm{P}=0.022)$ for mean HR. On average, HR was significantly lower with the KS treatment compared to PLA (mean $=148 \mathrm{bpm}$; mean $=152 \mathrm{bpm}$ ). Despite no change in $\mathrm{VO}_{2}$ during exercise, $\mathrm{HR}$ increased significantly at every time point of collection ( $\mathrm{P}$ $<0.05)$. HR data are shown in Figure 3. These findings indicate that the physical challenge had a significant effect on $\mathrm{HR}$ despite no change in $\mathrm{VO}_{2}$.

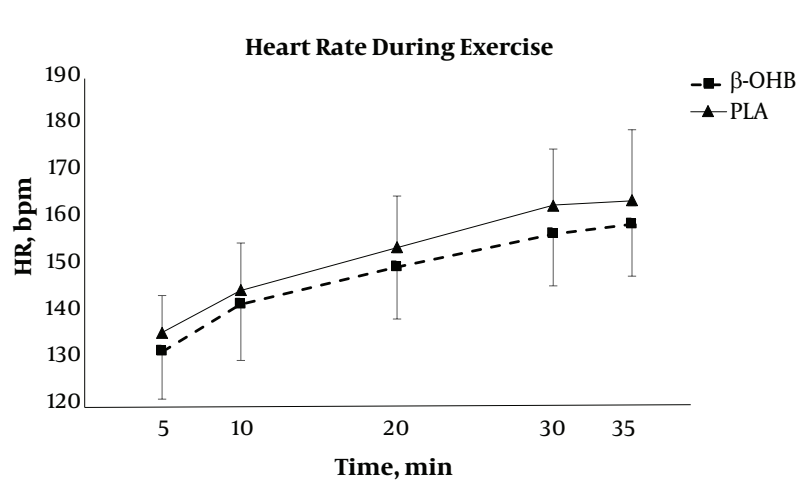

Figure 3. Changes in heart rate (HR) during exercise. Data are reported as mean \pm SD. HR was recorded 5, 10, 20, 30 and 35 minutes after the start of exercise. The 35 minutes time point was collected immediately upon completion of the test. $\beta$-OHB = beta hydroxybutyrate, PLA = placebo. A significant main effect for both time and treatment was found, with significantly lower $\mathrm{HR}$ in the $\beta$-OHB condition compared to PLA indicated by post hoc analysis.

\section{Discussion}

The primary finding of this study shows that steady state exercise at $60 \% \mathrm{VO}_{2 \text { peak }}$ in PPE resulted in significant OS that was not impacted by supplementing with KS for seven days. However, KS ingestion was associated with significant reductions in HR during exercise. Our findings are not in accordance with previous studies involving animals showing that ketone bodies reduce ROS and OS $(15,16,21)$. It is however important to note that this was the first study to investigate the impact of exogenous KS on markers of OS in humans.

Dietary interventions such as caloric restriction or the ketogenic diet have been suggested to facilitate metabolic and cardiovascular benefits, which may be related to increased antioxidant potential $(7,11)$. However, it is unclear if the reported health benefits are due to endogenous products of those diets (i.e., ketone bodies) or another mechanism. The exercise protocol caused OS which was indicated by a significant decrease in GSH levels immediately and 30-min post exercise. However, this study is limited by not including a non-loaded exercise condition to compare OS responses. It is therefore not clear if the amount of OS produced during this protocol is significantly greater than what would result from traditional (non-loaded) steady state exercise. A previous study (4) reported that treadmill exercise in FF wearing PPE resulted in greater OS compared to normal exercise clothes due to increased production of reaction oxygen species from increased mitochondrial strain. It is therefore assumed that the OS resulting from the current protocol is greater than what would result from traditional aerobic exercise (i.e., in normal clothes); however, this is merely speculation.

A review by Cotter et al. (22) suggests ketone bodies may act as signaling molecules to provide benefits to cardiovascular function by decreasing sympathetic tone and reducing HR. This may explain the findings of reduced HR during steady state exercise after ingesting KS for one week. These data are also supported by a finding from Kimura et al. (17) that reported reduced HR and sympathetic nervous system activity in caloric restricted mice that had elevated ketone levels. While the physiological responses to caloric restriction and increased ketone levels from exogenous intake are vastly different, it is important to note the suggestion from Cotter et al. (22) that ketones may act as signaling molecules to impact the sympathetic nervous system. The supplement also contained Ltaurine, L-leucine, and sodium which could have impacted these data. It is not likely that L-taurine would reduce HR since it has been shown to increase HR (23); however, the sodium content in the KS supplement could have impacted $\operatorname{HR}(24)$.

FF are more susceptible to OS compared to other occupations potentially due to metabolic stress from physiological and psychological stressors, in addition to poor dietary habits $(3,4,25)$. However, various occupational stressors can have an impact on biomarkers of OS which could 
be viewed as a limitation to the study. For instance, four of our FF responded to a structural fire within 48-hours prior to one of the testing sessions which could have impacted OS markers. Additionally, as previously mentioned, the KS supplement contained sodium, L-taurine and L-leucine which were not present in the placebo and may have impacted the HR data.

\subsection{Conclusions}

This was the first study to investigate the effects of exogenous KS ingestion on markers of OS in humans. Previous studies have suggested that $\beta$-OHB has anti-oxidative effects through various mechanisms; however, the current data do not suggest that exogenous KS reduce OS. It is perhaps possible that more than seven days of supplementation is such adaptations. In addition, the current protocol involved testing of a commercial supplement containing KS which are not as effective as ketone esters at increasing blood ketone levels (26). Our data support previous findings of reduced HR associated with KS; however, more mechanistic research is needed in order to determine if exogenous ketones can act as cardioprotective signaling molecules.

\section{Footnotes}

Authors' Contribution: Angelia Maleah Holland, Hunter Waldman, Harish Chander, and Matthew John McAllister, designed the study. Hunter Waldman, Steven Basham, Matthew John McAllister, and Harish Chander, performed the experiments. Angelia Maleah Holland, Hunter Waldman, Matthew John McAllister, JohnEric Smith, and Harish Chander, analyzed the data and drafted the initial manuscript. Hunter Waldman, Harish Chander, JohnEric Smith, Angelia Maleah Holland, Steven Basham, and Matthew John McAllister, edited and finalized the submitted manuscript.

Conflict of Interests: All authors report no conflict of interest associated with this manuscript.

Ethical Considerations: The study was approved by the University's Institutional Review Board.

Funding/Support: Financial support was provided by Pruvit Ventures, Inc., in addition to the Office of Research and Economic Development at Mississippi State University. Neither of these funding sources contributed directly in terms of study design, collection or analysis of data, writing of the manuscript, or decision to submit the manuscript for publication.

Patient Consent: Participants provided written informed consent.

\section{References}

1. Soteriades ES, Smith DL, Tsismenakis AJ, Baur DM, Kales SN. Cardiovascular disease in US firefighters: A systematic review. Cardiol Rev. 2011;19(4):202-15. doi: 10.1097/CRD.0b013e318215c105. [PubMed: 21646874].

2. Adetona O, Zhang JJ, Hall DB, Wang JS, Vena JE, Naeher LP. Occupational exposure to woodsmoke and oxidative stress in wildland firefighters. Sci Total Environ. 2013;449:269-75. doi: 10.1016/j.scitotenv.2013.01.075. [PubMed: 23434577].

3. Ferguson MD, Semmens EO, Dumke C, Quindry JC, Ward TJ. Measured pulmonary and systemic markers of inflammation and oxidative stress following wildland firefighter simulations. JOccup Environ Med. 2016;58(4):407-13. doi: 10.1097/JOM.0000000000000688. [PubMed: 27058482]. [PubMed Central: PMC4826715].

4. Park E, Lee YJ, Lee SW, Bang CH, Lee G, Lee JK, et al. Changes of oxidative/antioxidative parameters and DNA damage in firefighters wearing personal protective equipment during treadmill walking training.JPhys Ther Sci.2016;28(11):3173-7. doi:10.1589/jpts.28.3173. [PubMed: 27942144]. [PubMed Central: PMC5140824].

5. Ferraro E, Giammarioli AM, Chiandotto S, Spoletini I, Rosano G. Exercise-induced skeletal muscle remodeling and metabolic adaptation: Redox signaling and role of autophagy. Antioxid Redox Signal. 2014;21(1):154-76. doi: 10.1089/ars.2013.5773. [PubMed: 24450966]. [PubMed Central: PMC4048572].

6. Huang CJ, Webb HE, Zourdos MC, Acevedo EO. Cardiovascular reactivity, stress, and physical activity. Front Physiol. 2013;4:314. doi: 10.3389/fphys.2013.00314. [PubMed: 24223557]. [PubMed Central: PMC3819592]

7. Meydani M, Das S, Band M, Epstein S, Roberts S. The effect of caloric restriction and glycemic load on measures of oxidative stress and antioxidants in humans: Results from the CALERIE trial of human caloric restriction. J Nutr Health Aging. 2011;15(6):456-60. [PubMed: 21623467]. [PubMed Central: PMC3229089].

8. Pandey KB, Rizvi SI. Plant polyphenols as dietary antioxidants in human health and disease. Oxid Med Cell Longev. 2009;2(5):270-8. doi: 10.4161/oxim.2.5.9498. [PubMed: 20716914]. [PubMed Central: PMC2835915].

9. Hyatt HW, Kephart WC, Holland AM, Mumford PW, Mobley CB, Lowery RP, et al. A ketogenic diet in rodents elicits improved mitochondrial adaptations in response to resistance exercise training compared to an isocaloric western diet. Front Physiol. 2016;7. doi: 10.3389/fphys.2016.00533.

10. Kephart WC, Mumford PW, Mao X, Romero MA, Hyatt HW, Zhang $\mathrm{Y}$, et al. The 1-week and 8-month effects of a ketogenic diet or ketone salt supplementation on multi-organ markers of oxidative stress and mitochondrial function in rats. Nutrients. 2017;9(9). doi: 10.3390/nu9091019. [PubMed: 28914762]. [PubMed Central: PMC5622779].

11. Greco T, Glenn TC, Hovda DA, Prins ML. Ketogenic diet decreases oxidative stress and improves mitochondrial respiratory complex activity. J Cereb Blood Flow Metab. 2016;36(9):1603-13. doi: 10.1177/0271678X15610584. [PubMed: 26661201]. [PubMed Central: PMC5012517].

12. Wang $\mathrm{X}, \mathrm{Wu} \mathrm{X}$, Liu Q, Kong G, Zhou J, Jiang J, et al. Ketogenic metabolism inhibits histone deacetylase (HDAC) and reduces oxidative stress after spinal cord injury in rats. Neuroscience. 2017;366:3643. doi: 10.1016/j.neuroscience.2017.09.056. [PubMed: 29024787].

13. Kesl SL, Poff AM, Ward NP, Fiorelli TN, Ari C, Van Putten AJ, et al. Effects of exogenous ketone supplementation on blood ketone, glucose, triglyceride, and lipoprotein levels in Sprague-Dawley rats. Nutr Metab (Lond). 2016;13:9. doi: 10.1186/s12986-016-0069-y. [PubMed: 26855664]. [PubMed Central: PMC4743170]. 
14. Stubbs BJ, Cox PJ, Evans RD, Santer P, Miller JJ, Faull OK, et al. On the metabolism of exogenous ketones in humans. Front Physiol. 2017;8:848. doi: 10.3389/fphys.2017.00848. [PubMed: 29163194]. [PubMed Central: PMC5670148].

15. Shimazu T, Hirschey MD, Newman J, He W, Shirakawa K, Le Moan N, et al. Suppression of oxidative stress by beta-hydroxybutyrate, an endogenous histone deacetylase inhibitor. Science. 2013;339(6116):211-4. doi: 10.1126/science.1227166. [PubMed: 23223453]. [PubMed Central: PMC3735349].

16. Kim DY, Davis LM, Sullivan PG, Maalouf M, Simeone TA, Brederode $\mathrm{J}$, et al. Ketone bodies are protective against oxidative stress in neocortical neurons. J Neurochem. 2007;101(5):1316-26. doi: 10.1111/j.14714159.2007.04483.x.

17. Kimura I, Inoue D, Maeda T, Hara T, Ichimura A, Miyauchi S, et al. Short-chain fatty acids and ketones directly regulate sympathetic nervous system via G protein-coupled receptor 41 (GPR41). Proc Natl Acad Sci U S A. 2011;108(19):8030-5. doi: 10.1073/pnas.1016088108. [PubMed: 21518883]. [PubMed Central: PMC3093469].

18. Medicine ACoS. ACSM's guidelines for exercise testing and prescription. Lippincott Williams \& Wilkins; 2013.

19. Newman JC, Verdin E. Ketone bodies as signaling metabolites. Trends Endocrinol Metab. 2014;25(1):42-52. doi: 10.1016/j.tem.2013.09.002. [PubMed: 24140022]. [PubMed Central: PMC4176946].

20. Waldman HS, Basham SA, Price FG, Smith JEW, Chander H, Knight AC, et al. Exogenous ketone salts do not improve cognitive responses after a high-intensity exercise protocol in healthy college-aged males. Appl Physiol, Nutr Metab. 2018;43(7):711-7. doi:10.1139/apnm-2017-0724.
21. Maalouf M, Sullivan PG, Davis L, Kim DY, Rho JM. Ketones inhibit mitochondrial production of reactive oxygen species production following glutamate excitotoxicity by increasing NADH oxidation. Neuroscience. 2007;145(1):256-64. doi: 10.1016/j.neuroscience.2006.11.065. [PubMed: 17240074]. [PubMed Central: PMC1865572].

22. Cotter DG, Schugar RC, Crawford PA. Ketone body metabolism and cardiovascular disease. Am J Physiol Heart Circ Physiol. 2013;304(8):H1060-76. doi: 10.1152/ajpheart.00646.2012. [PubMed: 23396451]. [PubMed Central: PMC3625904].

23. Schaffer SW, Jong CJ, Ramila KC, Azuma J. Physiological roles of taurine in heart and muscle. J Biomed Sci. 2010;17 Suppl 1. S2. doi: 10.1186/1423-0127-17-S1-S2. [PubMed: 20804594]. [PubMed Central: PMC2994395].

24. Graudal NA, Hubeck-Graudal T, Jurgens G. Reduced dietary sodium intake increases heart rate. A meta-analysis of 63 randomized controlled trials including 72 study populations. Front Physiol. 2016;7:111. doi: 10.3389/fphys.2016.00111. [PubMed: 27047393]. [PubMed Central: PMC4805644].

25. Gaughan DM, Siegel PD, Hughes MD, Chang CY, Law BF, Campbell CR, et al. Arterial stiffness, oxidative stress, and smoke exposure in wildland firefighters. Am J Ind Med. 2014;57(7):748-56. doi: 10.1002/ajim.22331. [PubMed: 24909863]. [PubMed Central PMC4629844].

26. Evans M, Cogan KE, Egan B. Metabolism of ketone bodies during exercise and training: Physiological basis for exogenous supplementation. J Physiol. 2017;595(9):2857-71. doi: 10.1113/JP273185. [PubMed: 27861911]. [PubMed Central: PMC5407977]. 